\title{
Research on Navigation and Positioning Technology of Intelligent Accompanying Tool Car in Dispatching Automation Computer Room
}

\author{
Yufei Song ${ }^{1}$, Yu Shi ${ }^{1}$, Tianquan $\mathrm{Li}^{1 *}$, Xudong $\mathrm{Cao}^{1}$, Xiaohang $\mathrm{Liu}^{1}$, Li Sun ${ }^{1}$ and Manli Tang ${ }^{1}$ \\ ${ }^{1}$ State Grid Changchun Power Supply Company, Changchun, 130021, China
}

\begin{abstract}
In view of the wide variety of equipment in the dispatch automation machine room and the dense arrangement of cabinets, a navigation technology suitable for the dispatch automation machine room is proposed on the intelligent companion tool cart. In this paper, three sensors of ultrasonic, infrared and lidar are designed to form an intelligent neural sensor, and the information received by the intelligent neural sensor is calculated to generate a vector map through a software algorithm. At the same time, a plane coordinate network is established. The grid coordinate unit accuracy is $0.1 \mathrm{~cm}$. At the same time, the concept of "virtual fence" was put forward to fix the workers at the working point, which increased the safety of work. Subsequently, an experimental test of positioning and navigation of the tool cart was carried out. The experimental results showed that the tool cart can accurately locate and generate a vector map with an accuracy error of less than $10 \mathrm{~cm}$. The navigation method has a good application effect and has a good promotion value.
\end{abstract}

\section{Introduction}

With the continuous development of social economy and the continuous progress of science and technology, the power information system with the integration of digitization and high degree of information technology has become an important means of the existing smart grid. As a platform for seamless connection of various modules of the power system, the dispatching automation computer room is related to the safety and development of the entire power grid. With the continuous increase of various systems and equipment in the automated machine room, improving the efficiency of equipment operation and maintenance and safety management in the machine room has become an important issue[1,2]. In recent years, with the continuous development of navigation technology, intelligent substation operation and inspection robots have been widely used in the daily operation and maintenance of substations. However, there is a big difference between the dispatching automation machine room and the substation equipment. In view of the dense arrangement of cabinets in the automation machine room, no robots and other equipment with very suitable functions have been developed at this stage[3].

There are related products in domestic automated information computer room inspection robots, management robots, etc. There are many published documents and the research methods are relatively mature. However, the automated information computer room inspection robot is limited to the functions of equipment indicator light alarm, computer room environment, security, and personnel access management[4]. No automatic inspection robot has been developed. If similar equipment can be developed, it will greatly improve the efficiency and quality of automatic maintenance equipment, strengthen the safety management and control of computer room maintenance, and provide a strong guarantee for the company's safe production.

Precise positioning is the key technology of the robot in this project. It guarantees the realization of the navigation guidance of the operation point, the error prevention of the operation area, and the early warning of jailbreak in the operation and maintenance monitoring. Therefore, the research of navigation technology is particularly important $[5,6]$. At present, there are many domestic navigation equipment or software, but they do not use gridded vector diagrams, so when they are used in the computer room, the accuracy or accuracy is seriously affected. This article designs the navigation technology for the intelligent companion vehicle to make it specially suitable for shuttle navigation in the computer room. At present, none of the domestic navigation products adopts the product or application of this technology[7].

This paper uses landmark grid mapping technology to realize landmark grid mapping and precise positioning and error prevention of work sites, and more finely divide the locations that the navigation may reach, so that the navigation accuracy and accuracy are greatly improved. Especially for the automated machine room, the equipment and systems are placed in a regular

*Tianquan Li: 806306063@qq.com 
manner, which is suitable for establishing a finer grid map of the work site.

\section{Design of the overall structure of the navigation}

The equipment systems in the dispatch automation machine room are arranged in a regular manner, and the arrangement is very dense. According to the characteristics of the dispatch automation machine room, the navigation platform of the intelligent companion tool cart is set at the bottom of it, and the navigation platform is responsible for building a flat map and navigating. The physical map of the navigation platform is shown in Figure 1.

In response to the requirements of human-computer interaction, software programs need to be developed for navigation control. The software module uses standard $\mathrm{C}$ language to be developed under embedded Linux, the data communication uses standard RS232, TCP/IP and SQL, the navigation interface type is local area network, and the interface protocol is smart-ap2.0. The robot software is designed according to the $\mathrm{C} / \mathrm{S}$ method, the navigation software is named JFS-SMART1.0, the server software is embedded, and the client software runs on the control host or other computers.

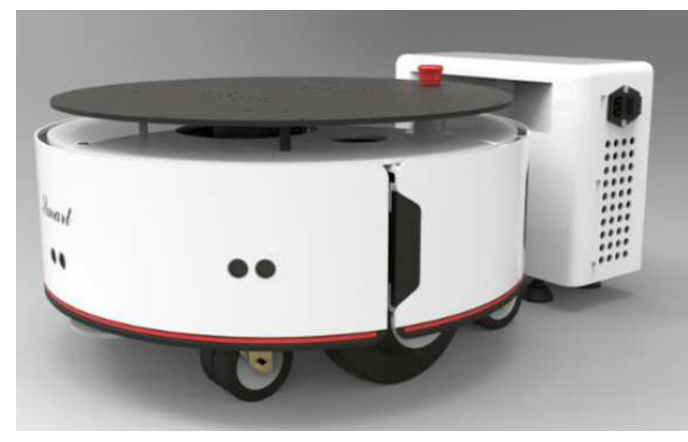

Fig. 1. Physical map of navigation platform

In application, the software first generates a plane map through the intelligent tool cart cruise memory trajectory, and then grids the map at centimeter level to construct a rasterized coordinate map. The gridded coordinate information and the map are stored in the map library of the navigation platform. The operation and maintenance task management software marks all possible operation points on the map as operation points, and all operation points are bound to the corresponding grid coordinates, and an operation and maintenance task is associated with one operation target point. When performing a task, the software submits the target point to the navigation platform, and the navigation platform autonomously navigates to the operation target point according to the network coordinates and brakes to stabilize the base. The specific navigation control process is shown in Figure 2.

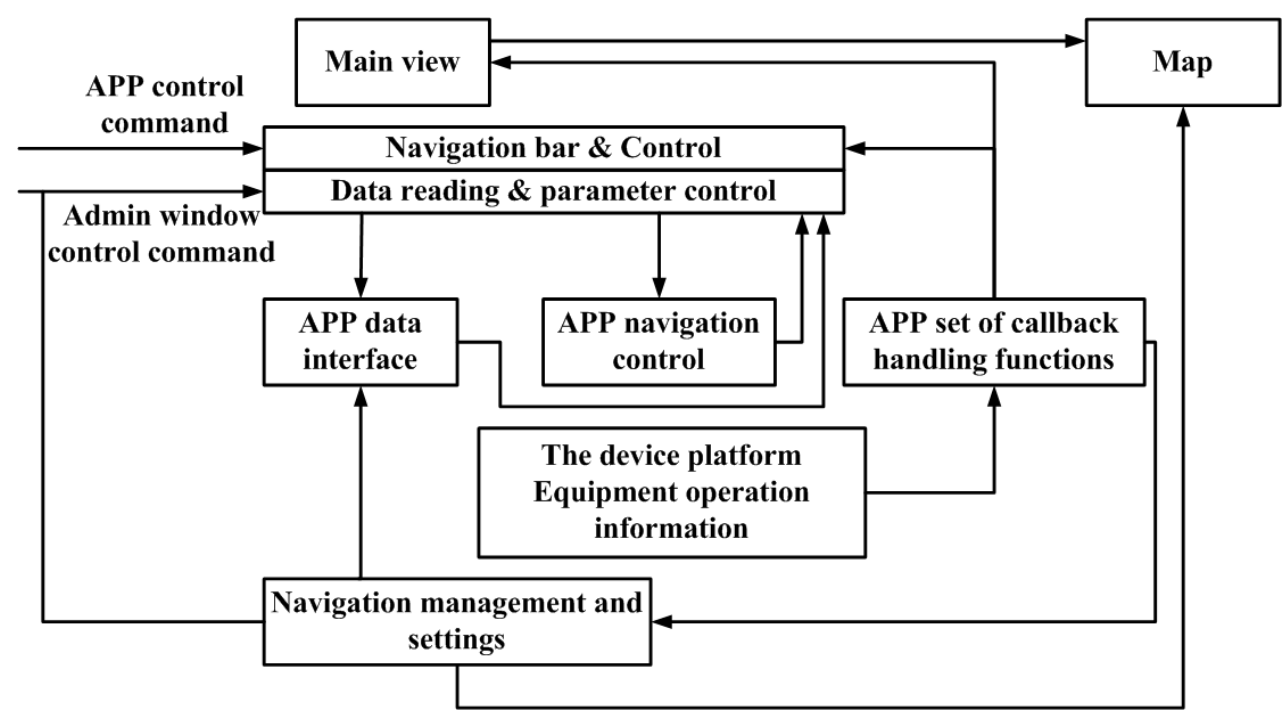

Fig. 2. Navigation control flow chart

\section{Design of navigation method}

\subsection{Design of the sensor}

The intelligent companion tool cart needs to realize automatic patrol and can lead the maintenance staff to the designated working place, so the tool cart needs to have the navigation and automatic movement function. Based on this functional requirement, the main hardware structure of the car mainly consists of a core control board, a motor, an obstacle avoidance navigation module, a speed measurement module, and wheels. Among them, the obstacle avoidance navigation module is the core module for automatic navigation. Its main functions are distance measurement and obstacle avoidance, portrait tracking, and its main components are infrared sensors, radar sensors, ultrasonic sensors and laser sensors. 


\subsection{Research on trajectory control and speed measurement method}

The walking trajectory of the intelligent companion tool cart is basically composed of the most basic trajectories, which completes the control of the basic trajectory, and also completes the control of the complex motion trajectory of the tool cart. This article divides the basic trajectory of the tool cart into two types, straight driving and turning. The straight-line driving control method is mainly controlled by a motor, which gives the tool wheel the same speed to ensure that it runs in a straight line. Turning control is to control the tool cart to rotate 90 degrees clockwise or counterclockwise, and the motor achieves the purpose of rotating by changing the rotation speed to give the wheels different speeds.

At the same time, when the tool cart is running, it is necessary to ensure that the tool cart's motion trajectory is accurate, so it is necessary to accurately control the traveling speed of the tool cart. In this article, the tool vehicle adopts the PWM speed regulation method, and the speed is adjusted by changing the width of the output pulse to ensure that the tool vehicle can travel at a stable speed to ensure the stability of navigation.

\subsection{Research on Positioning and Obstacle Avoidance Technology}

The ultrasonic sensor is installed on the chassis of the tool vehicle, and the transmitting probe of the ultrasonic sensor emits ultrasonic waves, and the ultrasonic waves travel in the air and return to the receiving device after encountering obstacles. By receiving the ultrasonic reflection signal emitted by itself, the propagation distance $\mathrm{S}$ is calculated according to the time difference between the ultrasonic emission and echo reception and the propagation speed, and the distance from the obstacle to the robot can be obtained.

According to the formula (1)

$$
S=T v / 2
$$

In the formula (1), $T$ - the time difference between ultrasonic transmission and reception; $v$ - the wave speed of ultrasonic propagation in the medium.

The positioning principle of the laser generator is similar to that of the ultrasonic wave. It emits a laser signal and calculates the distance based on the time difference of the signal reflected from the object. Then, determine the angle between the object and the transmitter according to the angle of the emitted laser, so as to obtain the relative position of the object and the transmitter.

The positioning principle of the infrared generator is slightly different from the above two types. The infrared IR marker emits modulated infrared rays, which are received by an optical sensor installed in the room for positioning.

The tool cart uses ultrasound, infrared, and lidar as intelligent neurosensors, and uses software algorithms to calculate vector maps from the signals received by multiple sensors. Establish a plane coordinate vector diagram according to the physical location of the computer room or other frequent operation points, with a grid coordinate unit accuracy of $0.1 \mathrm{~cm}$, and establish one or more vector diagrams as the navigation base map.

The system sets the starting origin on the vector map, and calculate the relative vector value of the movement through radar scanning and ultrasonic echo technology, so as to accurately locate. Several operation target points can be marked on the map, and the machine can be controlled to move from the origin to the target point to realize a plane grid navigation, and it can also reach another target point from one target point.

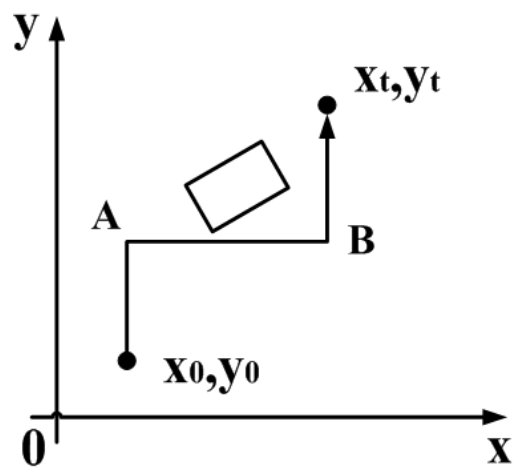

Fig. 3 Schematic diagram of obstacle avoidance

When there is an obstacle in the travel distance, the signal received by the intelligent neural sensor is used to determine the position of the obstacle, and the limit coordinates of the obstacle area are calculated, and the right side walking is the principle to avoid obstacles walking to reach the target location. The specific obstacle avoidance diagram is shown in Figure 3. When the tool cart takes $\left(\mathrm{x}_{0}, \mathrm{y}_{0}\right)$ as the starting point and at the same time judges the coordinate limit of the obstacle, it turns around the obstacle at points $\mathrm{A}$ and $\mathrm{B}$ to reach the target position $\left(\mathrm{x}_{\mathrm{t}}, \mathrm{y}_{\mathrm{t}}\right)$.

\subsection{Design of virtual wall}

The "virtual fence" refers to the concept of "pull the fence and hang the red curtain" in the operation of the power system. The function of the virtual fence is similar to the fine and dynamic division of the map area. The laser radar must be equipped to achieve reliable accuracy, and the "virtual fence" can be realized by using the laser radar ranging function.

A boundary line can be set up for the whole map or a partial area of the map generated by the tool cart scanning. This boundary line is a virtual wall for movement control. The virtual wall is like a physical barrier wall for the tool cart. The tool cart can only move inside the wall, and at the same time, the operator can only move inside the wall. As shown in Figure 4, when the tool cart travels to the $\left(\mathrm{x}_{0}, \mathrm{y}_{0}\right)$ operating point, it will form a square area in the surrounding $\pm t$ area. When the operation and maintenance personnel work beyond the current area, the tool cart laser radar device will be triggered to send out a warning signal, and the software can make corresponding processing according to the warning signal. That is, when the operator's abscissa 
received by the tool cart is less than $\mathrm{x}_{0}-\mathrm{t}$ or greater than $\mathrm{x}_{0}+\mathrm{t}$; when the ordinate is less than $\mathrm{y}_{0}-\mathrm{t}$ or greater than $\mathrm{y}_{0}+\mathrm{t}$, a warning signal is issued.

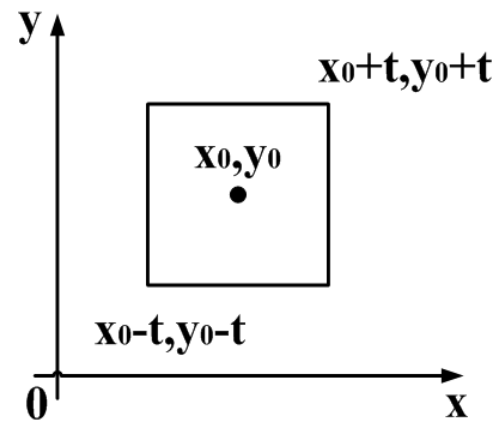

Fig. 4 Schematic diagram of virtual fence

\section{Experimental test of vector navigation and positioning system}

In order to verify the accuracy of the tool cart navigation map generation, six different geographic regions were selected to test the tool cart vector mapping function. The test results are shown in Table 1.

Table 1. Vector map generates test results

\begin{tabular}{|c|c|}
\hline location & Vector map \\
\hline office & $\mathrm{Y}$ \\
\hline Dispatch automation room & $\mathrm{Y}$ \\
\hline corridor & $\mathrm{Y}$ \\
\hline UPS & $\mathrm{Y}$ \\
\hline Battery room & $\mathrm{Y}$ \\
\hline meeting room & $\mathrm{Y}$ \\
\hline
\end{tabular}

As shown in the above table, the tool cart can generate vector maps in the above six geographic environments. It can be seen that the tool cart can reliably perform vector mapping work.

Then seven operating points were selected to test whether the tool cart is accurately navigated and the amount of travel error. The results are shown in Table 2 below.

Table 2. Navigation and positioning test results

\begin{tabular}{|c|c|c|}
\hline & Error $/ \mathrm{m}$ & $\begin{array}{c}\text { Obstacle } \\
\text { avoidance }\end{array}$ \\
\hline Sta10-1 & $0.08 \mathrm{~m}$ & Y \\
\hline \#1UPS & $0.02 \mathrm{~m}$ & Y \\
\hline \#2air conditioner & $0.01 \mathrm{~m}$ & Y \\
\hline Satellite clock & $0.05 \mathrm{~m}$ & Y \\
\hline Disk array & $0.09 \mathrm{~m}$ & Y \\
\hline Switches & $0.08 \mathrm{~m}$ & Y \\
\hline $\begin{array}{c}\text { Power distribution } \\
\text { cabinet }\end{array}$ & $0.03 \mathrm{~m}$ & $\mathrm{Y}$ \\
\hline
\end{tabular}

Draw an error graph based on the test results in the table, as shown in Figure 5.

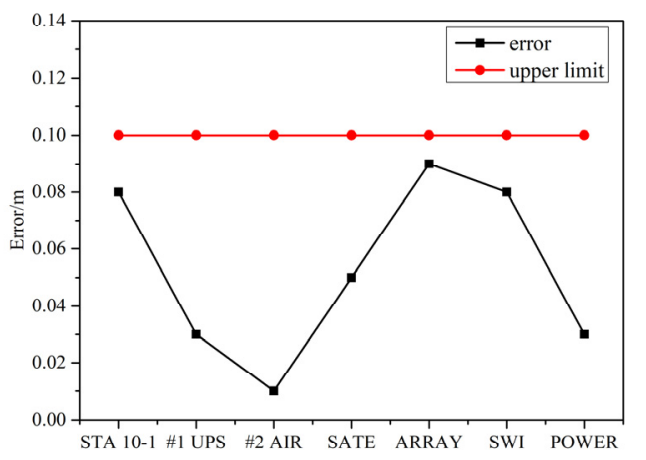

Fig. 5 Navigation and positioning test error diagram

As shown in Figure 5, the tool cart can accurately reach the designated working place, and the accuracy error is less than $10 \mathrm{~cm}$.

In summary, the tool cart can accurately carry out grid mapping of landmarks and precise positioning and error prevention of work sites, and the accuracy error meets the requirements.

\section{Conclusion}

Positioning and navigation are important technical content of the application and development of artificial intelligence. This paper studies the navigation technology of the intelligent companion vehicle, and through experimental tests, it is found that the tool vehicle can accurately generate a gridded vector map, and can achieve more accurate indoor positioning, with a test error of $<10 \mathrm{~cm}$. The specific research content is as follows:

(i) This article designs the overall structure of the navigation of the intelligent companion tool cart. Install ultrasonic, infrared and lidar as sensors on the chassis of the tool cart, and design and develop software and interfaces;

(ii) This article explores the navigation trajectory control and speed measurement methods of the tool vehicle. The complex trajectory of the tool vehicle is divided into two ways: straight driving and turning, and the speed of the tool vehicle is controlled by changing the PWM of the motor;

(iii) This article explores positioning and obstacle avoidance technology. The combination of ultrasonic, infrared, and lidar sensors is used as an intelligent neurosensor, and a vector diagram with a unit accuracy of $0.1 \mathrm{~cm}$ is used as a navigation base map using software algorithms, and it can be used for obstacle avoidance movement;

(iv) In this paper, a "virtual fence" technology is designed. By calculating the vector map coordinates of a fixed operating point, the working area is delineated, and the operating personnel are supervised through the intelligent nerve sensor to ensure the safety of the operation. 


\section{References}

1. HX, Lin. Environmental monitoring system of data center. Intelligent Building and City Information, 5, 59-62. (2009)

2. EM, Zhao. Research on the motion control of intelligent inspection robots. Proceedings of the 14th Shenyang Science Conference Science, Agriculture, Medicine.1025-1028, (2017).

3. Zhu A, Tu Y, Zheng W. Design and Implementation of High-Voltage Transmission Line Inspection and Foreign Bodies Removing Robot. 852-856, (2018)

4. Hongling Han, Fenglei Yang. Path planning of an indoor mobile robot navigated by infrared. International Conference on E-Product E-Service and E-Entertainment. Zhengzhou. 1-4. (2010)

5. L Song, H Wang, P Chen. Automatic Patrol and Inspection Method for Machinery Diagnosis Robot. Sound Signal-Based Fuzzy Search Approach. IEEE Sensors Journal, 2020.

6. Kwon Y S. Design and Motion Planning of a TwoModule Collaborative Indoor Pipeline Inspection Robot. IEEE Transactions on Robotics, 28(3):681696, (2012).

7. Tian Guoguo, Wang Jiachao, Duan Peng. Research on obstacle avoidance technology of ward patrol robot in complex environment. Journal of Huazhong University of Science and Technology (Natural Science Edition), 041:312-315, (2013). 